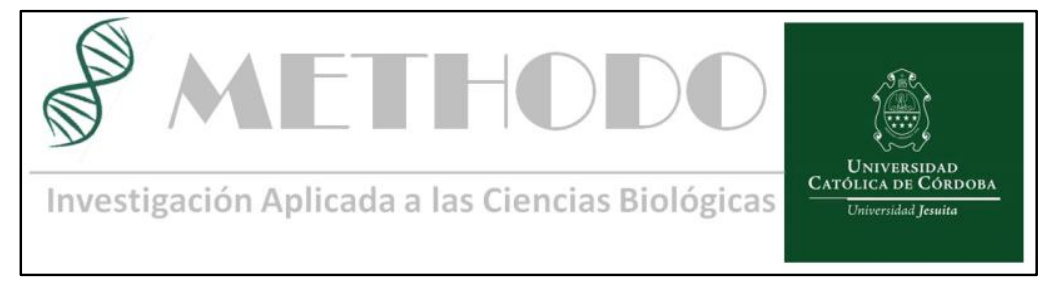

\title{
Infiltración versus remineralización: Estudio preliminar ex-vivo de alternativas para el tratamiento no invasivo de lesiones por caries con materiales bioactivos
}

\section{Caries infiltration versus remineralization: A preliminary ex- vivo study of non-invasive alternative treatments using bioactive materials}

\author{
Od. Martín Sainz Aja ${ }^{1}$, Od. Laura Isabel Ribba ${ }^{2}$, Dr. Ricardo Juan Cabral ${ }^{3}$, Dr. Gustavo Fabián Molina ${ }^{1}$ \\ ${ }^{1}$ Cátedra de Materiales Dentales y Biomateriales, Carrera de Odontología, Facultad de Ciencias de la Salud, Universidad Católica \\ de Córdoba. ${ }^{2}$ Cátedra de Microbiología y Cardiología, Carrera de Odontología, Facultad de Ciencias de la Salud, Universidad \\ Católica de Córdoba. ${ }^{3}$ Cátedra de Materiales Dentales, Facultad de Odontología, Universidad Nacional de Córdoba
}

\section{Resumen:}

El objetivo fue determinar profundidad de penetración en dentina cariada artificial y cambios en su dureza superficial de dos protocolos de tratamiento: 1- infiltración del tejido cariado con resina fluida modificada con nanopartículas de óxido de cinc y 2- remineralización con fluoruro di-amino de plata. Para tal fin se prepararon láminas de terceros molares sanos de $4 \mathrm{~mm}$ de espesor en los cuales se generaron lesiones artificiales de caries sobre la superficie oclusal de la muestra, en un área de 6x6 mm. Las muestras se dividieron en dos grupos de tratamiento. Cada muestra fue seccionada en tres porciones de superficie cariada y una sección adicional de dentina intacta. En dos de las secciones con caries se aplicó el protocolo de tratamiento correspondiente. De esas dos secciones, una fue utilizada para realizar un mapeo en microscopio electrónico de barrido (MEB), identificando la presencia de plata $(\mathrm{Ag})$ o cinc $(\mathrm{Zn})$. En la porción restante que recibió tratamiento se evaluó su dureza superficial y se la comparó con la parte que no recibió tratamiento y con la sección adicional de dentina intacta. La dureza superficial se valoró mediante diagramas de fuerza obtenidos por un microscopio de fuerza atómica (AFM). Los resultados evidenciaron una penetración significativa de Ag y presencia superficial de $\mathrm{Zn}$ en las muestras. Los diagramas de fuerza mostraron los valores más elevados en las superficies tratadas con Fluoruro de plata, seguidas por la dentina intacta y las superficies que recibieron la resina con nanopartículas de cinc. La dentina cariada presentó valores asociados a menor dureza. Se concluyó que ambos tratamientos pueden mejorar las propiedades mecánicas superficiales del tejido, pero se necesitan estudios más específicos para determinar la profundidad de penetración en dentina afectada por caries.

\begin{abstract}
:
The aim was to determine the penetration depth into caries-affected dentin and the surface hardness of two treatment protocols: 1- caries infiltration using fluid resin modified by nanosized cinc particles and 2- remineralization by means of topical diamine silver fluoride. For that purpose, $6 \times 6 \mathrm{~mm}$ artificial caries lesions were produced in occlusal surfaces of $4 \mathrm{~mm}$-thick slices obtained from sane third molars. Tooth samples were divided into the two treatment groups. Each sample was split into three caries-affected sections and one non-affected additional portion. Treatment protocols were applied in two of these cariesaffected sections, sending one of them for mapping of silver $(\mathrm{Ag})$ or cinc $(\mathrm{Zn})$ on a scanning electron microscope. Surface hardness was assessed in the other treated section and compared to the results
\end{abstract}


obtained from the others sections of the same sample by means of force mapping produced by an Atomic Force Microscope (AFM). Results showed a significant penetration of $\mathrm{Ag}$ into the body of the lesión whereas $\mathrm{Zn}$ was only found on the surface. Force mapping expressed higher surface hardness on sections treated with silver fluoride, followed by those non-affected and the ones treated with resin modified with cinc nanoparticles. Lowest values were observed in caries-affected surfaces. It was concluded that both treatments may increase mechanical properties in the surface of the lesions although more sensible instruments are needed to determine the penetration depth of the products tested in this preliminary study.

\section{Introducción:}

La aplicación de los principios de adhesión fue un punto de inflexión en la Odontología Restauradora de tal modo que los paradigmas que dominaban el abordaje de las lesiones por caries se fueron reformulando, con el consecuente desarrollo de nuevos materiales y tecnologías orientadas a la máxima conservación de los tejidos dentarios.

Estos avances, respaldados por una mejor comprensión de la etiopatogenia de la enfermedad de caries, estimularon acciones preventivas para minimizar los factores de riesgo relacionados a la susceptibilidad del diente a ser afectado por productos de degradación bacteriana. En tal sentido, la aplicación de fluoruros y el uso de selladores para bloquear surcos, fosas y fisuras, se constituyeron en las principales estrategias para aumentar la resistencia de las estructuras del diente amenazadas por la patología.

Cuando la progresión de la lesión de caries supera estas barreras preventivas, la pérdida de minerales y la desnaturalización de los componentes orgánicos del diente generan tejidos frágiles y reblandecidos que, eventualmente provocan la formación de una cavidad. Consecuentemente, el tratamiento sintomático de esa lesión se corresponde con la remoción de las estructuras comprometidas por el avance de microorganismos y sus productos metabólicos, para luego devolver forma y función a través de sustitutos biocompatibles que rellenan la cavidad resultante. La tendencia actual para restaurar estas lesiones procura no sólo preservar las estructuras que resulten indemnes al proceso de limpieza cavitaria sino más aún, estimular la reparación a partir de la activación de respuestas biológicas. El aporte de la ingeniería tisular para el desarrollo de materiales biomiméticos o bioactivos permite avizorar un futuro en el que la regeneración de tejidos y estructuras extracelurares como el esmalte dental, recuperen las propiedades mecánicas perdidas por la degradación generada durante el proceso carioso. Para tal fin, es necesario anular la actividad bacteriana en el cuerpo de la lesión de caries, devolver elementos estructurales de los tejidos afectados (células, proteínas y minerales) y aportar aquellos que brinden sustentabilidad al proceso de reparación. ${ }^{1}$

En este sentido, la intervención en estadios iniciales de la lesión por caries se ha fundamentado en la intención de remineralizar el esmalte afectado por el ataque ácido, devolviéndole calcio y fosfato a los cristales de hidroxiapatita, además de la incorporación de flúor para apuntalar su resistencia a la disolución. ${ }^{2}$ No obstante, si bien la capacidad de penetración de estos iones y su eficacia depende de las diferentes formulaciones químicas, concentraciones y modo de aplicación, la barrera natural que supone las características histológicas del esmalte es un obstáculo de consideración.

Otra estrategia propuesta para la recuperación del esmalte no cavitado es la infiltración de lesiones mediante el uso de polímeros resinosos, posterior a un tratamiento superficial con ácido clorhídrico para aumentar la permeabilidad del sustrato infiltrado. ${ }^{3}$ Este recurso ha sido objeto de estudios con resultados positivos en lesiones superficiales aunque presenta como elemento de discusión que sólo se limitaría a enmascarar fenómenos ópticos de traslucidez, sin aportar elementos reconstituyentes ni promover respuestas defensivas del diente frente al eventual avance de una lesión. ${ }^{4}$

La incorporación de nanotransportadores (nanocarriers) en sistemas adhesivos a base de resinas metacrílicas con la finalidad de inhibir la acción de metaloproteasas en la interfaz adhesiva demuestra la posibilidad de utilizar polímeros como vehículos para la introducción de elementos bioactivos a través de sustancias infiltrantes. ${ }^{5}$ Las nanopartículas a base de cinc transportadas para bloquear las MPP en la degradación de la matriz orgánica dentinaria también se han incorporado a la formulación de cementos de ionómero vítreo para apuntalar sus propiedades químicas y mecánicas. ${ }^{6}$

De este modo, la combinación de los conceptos de infiltración y transporte de nanopartículas bioactivas se analiza en el presente trabajo como una opción novedosa para el tratamiento no invasivo de lesiones por caries, en comparación con un sistema de remineralización basado en la aplicación de una solución de fluoruro di-amino de plata al $38 \%$, utilizada con buenos resultados clínicos para detener la progresión de lesiones tanto en esmalte como en dentina. ${ }^{7-8}$ El objetivo del estudio fue comparar la capacidad de infiltración y la dureza superficial de caries artificiales en 
dentina tratadas con los dos tratamientos mencionados.

\section{Materiales y métodos:}

La capacidad de infiltración y la dureza superficial de dentina afectada por caries y luego tratada con las sustancias remineralizadas e infiltrantes se evaluó en 8 terceros molares sanos del Banco de Dientes Humanos de la Facultad de Odontología, Universidad Nacional de Córdoba (res. HCD 333/17). Las muestras para experimentación se obtuvieron por cortes de la porción coronaria paralelos entre sí y perpendiculares al eje longitudinal del diente, generando láminas de 4 mm de espesor.

Se cubrió la totalidad de las superficies de cada lámina con esmalte de uñas (Revlon), con excepción de una superficie cuadrangular de 6x6 $\mathrm{mm}$ en una de sus caras para generar lesiones artificiales de caries exponiendo la superficie libre a una solución de $\mathrm{C} 3 \mathrm{H} 6 \mathrm{O} 30.1 \mathrm{M}$ durante 72 horas a pH 4.5 almacenadas a $37^{\circ} \mathrm{C}$.

De cada lámina se seccionaron 4 porciones iguales, 3 correspondientes a la zona cariada y 1 que había quedado recubierta por esmalte para preservar la dentina de la lesión artificial de caries. Del total de las muestras se asignaron la mitad a cada grupo de tratamiento. En el esquema de la (Figura 1) se describe la obtención, distribución y tratamiento de las muestras.

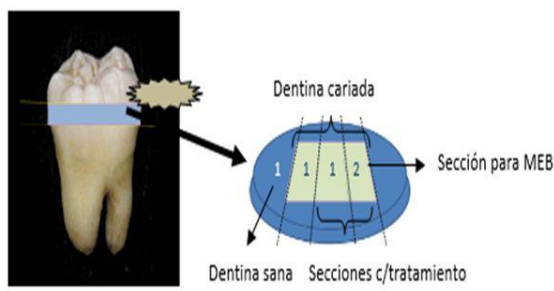

Ref. 1- Secciones analizadas con Microscopio de Fuerzas Atómicas; 2- Sección observada con Microscopio Electrónico de Barrido

Figura 1- Esquema sobre la obtención y distribución de las secciones de cada muestra

Después de incluirlas en resina acrílica se aplicó el protocolo de tratamiento en dos de las tres secciones afectadas por caries para luego evaluar dureza superficial y capacidad de infiltración respectivamente. A las porciones restantes (1 con caries y otra sin lesión) se las utilizó para determinar diferencias en la dureza superficial en una misma muestra, comparando la dentina sana, la afectada por caries y el efecto de los tratamientos en la dureza de la dentina cariada.

Grupo A: Sistema de infiltración resinoso
Para la obtención de nanocristales se propuso el uso de la homogenización de alta presión (HAP), surgida en la década del 90 cuando Müller et al puso a punto esta técnica de desintegración para la producción de nanocristales ${ }^{16}$ y para la obtención de nanopartículas lipídicas con el uso o no de temperatura. Se seleccionaron nanopartículas de óxido de cinc ( $\mathrm{ZnO} @ \mathrm{NP}, 50 \mathrm{~nm})$.

Los sistemas obtenidos fueron caracterizados mediante microscopia electrónica de transmisión y barrido (TEM, SEM), difracción de rayos $\mathrm{X}$ (XRD), análisis térmico (DSC-TG), espectroscopia FTIR-DRIFTS, determinación de tamaño de partícula (light scattering) y carga en superficie (potencial Z).

La estabilidad de las nanopartículas fue medida cinéticamente en medios de cultivo y en relación al polímero utilizado como vehículo para infiltración de lesiones cariosas, el trietilenglicol dimetacrilato (resina IconR, DMG Alemania).

La proporción de nanopartículas de cinc en relación a la resina fluida fue de $1.2 \mu \mathrm{g}$ de óxido de cinc por $1 \mathrm{ml}$ de trietilenglicol dimetacrilato.

Previo a la aplicación del producto infiltrante, se realizó la preparación de la superficie a tratar según el protocolo del fabricante de Icon, mediante la colocación de un gel de ácido clorhídrico al 15\% durante 15 segundos. A contuación, se lavó con agua destilada, se secó la superficie con aire limpio y se aplicó un solvente a base de acetona para aumentar la energía superficial por otros 15 segundos. Las lesiones fueron infiltradas con la combinación del sistema resinoso Icon y las nanocápsulas lipídicas cargadas con cinc. La resina infiltrada fue fotopolimerizada durante 60 segundos y los especímenes fueron almacenados durante 48 horas a $37^{\circ} \mathrm{C}$.

Grupo B: Fluoruro di-amino de plata - Ag(NH3)2F La aplicación de este producto sobre las lesiones de caries no requierió de un pre-tratamiento superficial más allá de la limpieza con agua destilada y el secado con aire limpio. Se utilizó fluoruro de plata en una concentración de $38 \%$ de una marca comercial con fabricación local (Fagamin, Tedequim, Córdoba - Argentina), frotando el líquido con un micro-aplicador (Microbrush, 3M Espe, St. Paul, Estados Unidos) durante 10 segundos. Los especímenes fueron almacenados durante 48 horas a $37^{\circ} \mathrm{C}$.

\section{Evaluación de la profundidad de infiltración}

De cada muestra se obtuvo una sección de dentina con caries artificial que recibió alguno de los tratamientos evaluados, observándola al microscopio de manera tal que pudiera rastrearse la penetración de los productos terapéuticos en sentido ocluso-apical. 
La presencia de plata o zinc en cada muestra se detectó empleando un espectrómetro dispersivo de energía (EDS) acoplado al microscopio electrónico de barrido FE-SEM iigma. Condiciones experimentales: $\mathrm{EHT}=20 \mathrm{keV}$, Apertura $=60 \mu \mathrm{m}$ )

\section{Evaluación de la dureza superficial}

Se separaron tres secciones de cada diente de los dos grupos experimentales: una sección de dentina sana, que había sido protegida con esmalte durante la generación de caries artificial; una sección a la cual no se le aplicó el tratamiento correspondiente; una tercera sección de diente que recibió tratamiento. De este modo se pretendió comparar en la misma muestra los valores previo y posterior a la aplicación de los productos terapéuticos en relación a la dentina intacta de esa pieza.

La dureza superficial se determinó por medio de diagramas de fuerza obtenidos a través de un microscopio de fuerza atómica (AFM, Departamento de Físico-Química, Facultad de Ciencias Químicas, UNC), el cual realizó mapas bidimensionales de curvas de fuerza, elasticidad, adhesión y deformación registradas de las superficies evaluadas.

\section{Resultados:}

Los mapeos elementales mostraron que el catión Ag penetró en las 4 muestras tratadas con Fluoruro di-amino de plata al $38 \%$ en un promedio de 135 (45) $\mu \mathrm{m}$ mientras que el $\mathrm{Zn}$ rastreado en las muestras que recibieron el polímero de infiltración modificado con nanopartículas de cinc no superó valores medios de penetración de 15 (6) $\mu \mathrm{m}$. (Figura 2,3 y 4).

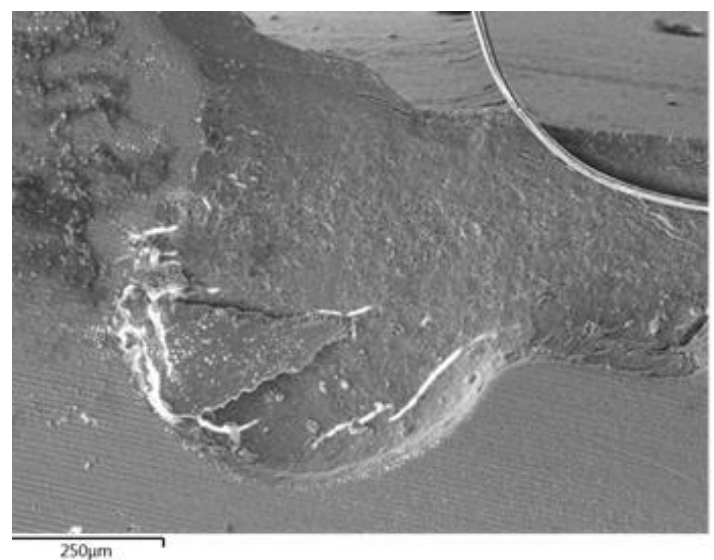

Figura 2- Vista sagital de una lesión artificial de caries en microscopio electrónico de barrido (MEB)

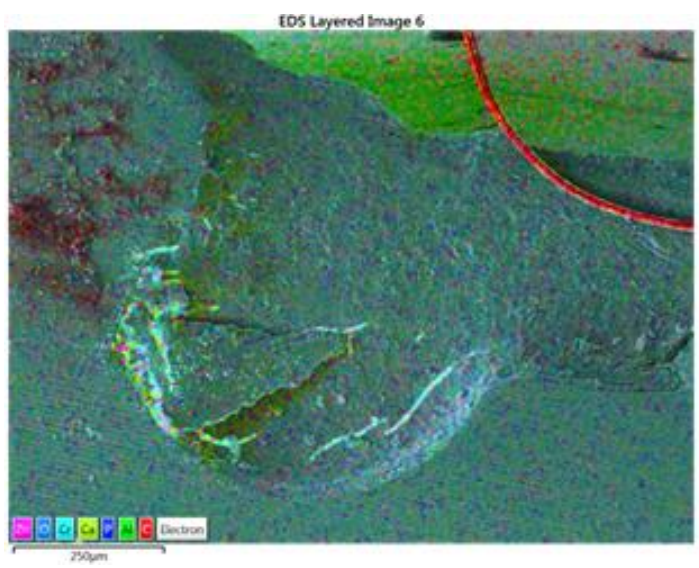

Figura 3- Vista sagital de una lesión de caries infiltrada por resina fluida modificada con nanopartículas de cinc analizada en el MEB con EDS

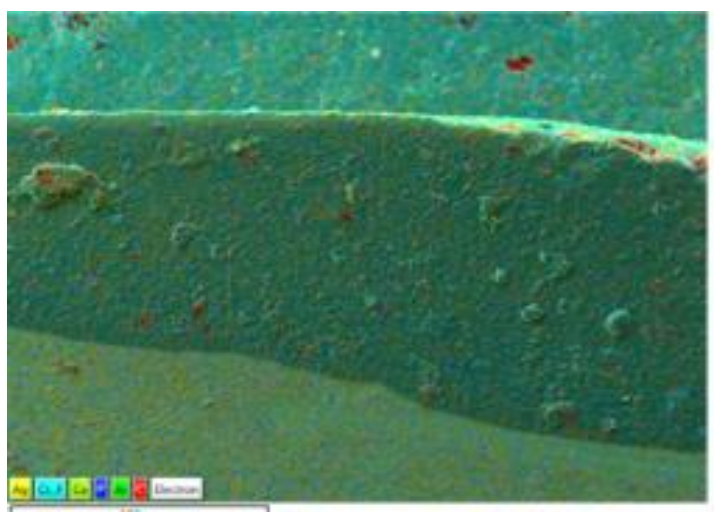

Figura 4- Vista sagital de una lesión de caries tratada con Fluoruro di-amino de plata al $38 \%$ analizada en el MEB con EDS.

Los diagramas de fuerza obtenidos por medio del microscopio de fuerza atómica generaron curvas de carga y desplazamiento en las cuales se evidencia una mayor dureza en las secciones de las muestras con dentina sana y con dentina tratada con los dos protocolos terapéuticos, en comparación con las secciones afectadas por caries que no recibieron tratamiento. (Figura 5)
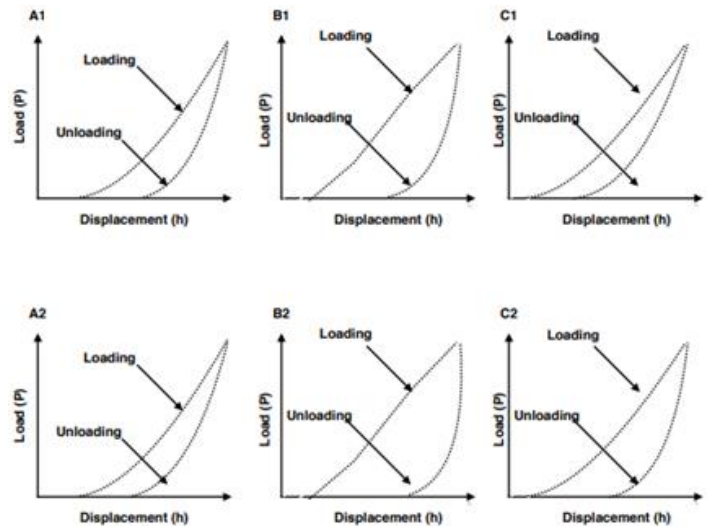

Figura 5- Diagramas de fuerza obtenidos por el microscopio de fuerza atómica (AFM)

Revista Methodo: Investigación Aplicada a las Ciencias Biológicas. Universidad Católica de Córdoba. Jacinto Ríos 571 Bo Gral. Paz. X5004FXS. Córdoba. Argentina. Tel.: (54) 3514517299 / Correo: methodo@ucc.edu.ar / Web: methodo.ucc.edu.ar |ARTICULO ORIGINAL Methodo 2018;3(3):52-58 
Ref. A1 y A2 Secciones de dentina sana; B1 y B2 Secciones de dentina afectada por caries; C1 Secciones de dentina cariada tratada con Fluoruro di-amino de plata al 38\%; C2 Secciones de dentina cariada tratada con Resina fluida modificada con nanopartículas de cinc.

\section{Discusión:}

Si bien el objetivo del presente estudio fue evaluar la penetración de los productos terapéuticos en el tejido afectado por caries y la modificación de su dureza superficial, el carácter preliminar del trabajo permitió al mismo tiempo poner a punto la metodología para determinar el comportamiento de las variables estudiadas en las muestras y establecer su pertinencia.

La posibilidad de localizar con eficacia el elemento Ag en la profundidad de las lesiones fue contundente, en coincidencia con otros estudios ex-vivo donde también se alcanzaron $150 \mu \mathrm{m}$ de penetración. ${ }^{9}$ Sin embargo, los resultados del mapeo de cinc en las muestras tratadas con el producto experimental a base de resina modificada con nanopartículas fueron insuficientes para determinar la profundidad de penetración, identificando el elemento $\mathrm{Zn}$ sólo en pequeñas cantidades a nivel superficial de la muestra. En este caso, es posible que la concentración de nanopartículas en relación a la resina resultara por demás escasa para lograr una distribución adecuada, llegando a ser prácticamente imperceptible por el instrumento.

Por otra parte, la utilización del microscopio de fuerza atómica se constituyó en un recurso de gran valor para el manejo de muestras biológicas de tamaño reducido, como opción al microdurómetro tradicionalmente empleado para evaluar dureza Vickers. El AFM, entre varios usos, es una herramienta para dirigir el diseño de estructura de andamiaje en ingeniería tisular. ${ }^{10}$ En este caso, la simulación de una nanoindentación sobre la superficie derivó de la aplicación de fuerzas atómicas que contrastaban con las respuestas viscoelásticas y plásticas del tejido, generando una curva espectroscópica donde se expresaban las diferentes consistencias de la superficie.

Para la realización de este estudio se consideró el diseño de nanocápsulas lipídicas (LNC) las cuales han surgido como una alternativa a los sistemas portadores de liberación modificada y/o direccionada de moléculas bioactivas (nanopartículas poliméricas, liposomas) debido a las numerosas ventajas que ofrecen comparadas a otros sistemas desarrollados. ${ }^{11} \mathrm{El}$ agente activo, en este caso las nanopartículas de óxido de cinc, podría ser liberado en forma gradual como consecuencia de la erosión (degradación enzimática) o por difusión desde las partículas. Estos procesos son regulados por la naturaleza del lípido, tamaño de partícula, tipo y cantidad de surfactante, y método de elaboración. La dificultad en dicha regulación fue determinante para optar por la incorporación de nanocristales a granel, como experiencia preliminar, considerando el vehículo utilizado para transportar estas nanopartículas. ${ }^{12-13}$

Este aspecto se constituyó en un nuevo desafío. La utilización de un polímero diseñado para infiltración de caries demostró ser el medio más estable para alojar las nanopartículas de óxido de cinc. ${ }^{14}$ Este sistema incluye además una preparación de la superficie a infiltrar que promueve una mayor permeabilidad del sustrato, procurando la mejor penetración del infiltrante. ${ }^{15}$ Para el desarrollo de un producto que promueva la regeneración tisular de un diente afectado por la progresión de una lesión de caries, el investigador se enfrenta con una serie de condicionantes como la heterogeneidad de las estructuras que requieren reparación, representadas en los diferentes sustratos histológicos de un diente: a nivel superficial, el esmalte, que no es un tejido propiamente dicho sino un estructura extracelular de minerales; luego la dentina, una red de colágeno mineralizada y atravesada por túbulos que contienen prolongaciones celulares desde la pulpa; por último, la pulpa, un paquete de vasos y células que mantienen el diente con sensibilidad y nutrición. Resultaría más factible procurar dicha regeneración separando cada sustrato que intentarlo todo en un solo procedimiento.

De hecho, la revascularización de la pulpa en elementos permanentes jóvenes ha representado un avance significativo en casos de traumatismos dentarios, reportándose numerosas publicaciones que avalan estos procedimientos. Es relevante la utilización de células indiferenciadas para la neoformación del tejido pulpar. ${ }^{16}$

La hipótesis de reparación en esmalte y dentina supone la utilización de las estructuras afectadas como un andamiaje que alojará los elementos que se perdieron durante la progresión de la lesión de caries, además de otros que pudieran adicionarse para potenciar algunas propiedades mecánicas y biológicas en el proceso de cicatrización de la lesión.

En el esbozo de este estudio se inicia el camino para lograr un producto bioactivo con las propiedades de reparación deseables. Como primer paso, es fundamental lograr impregnar la totalidad del tejido afectado para reconvertirlo. Los resultados preliminares muestran que en el material experimental a base de resina $y$ nanopartículas no es posible identificar cinc más allá de la superficie, dejando planteada la discusión sobre si se trata de su escasa capacidad de 
infiltración o la dificultad de rastrear bajas concentraciones de partículas de por sí extremadamente pequeñas.

A pesar de estas observaciones, el producto experimental logró aumentar la dureza superficial de las lesiones, probablemente potenciado por la polimerización del componente resinoso.

En cuanto a los resultados más alentadores obtenidos en el grupo de tratamiento del Fluoruro de plata, éstos se corresponden con los reportes que avalan la capacidad de re mineralización insitu de lesiones activas, adjudicando a este producto la

doble acción de formación de fluor-hidroxiapatita y el potencial antibacteriano del ion plata. ${ }^{7-8}$

Una vez alcanzadas las expectativas de infiltración total del tejido afectado por caries, será posible avanzar sobre nuevas sustancias remineralizantes, antibacterianas y/o promotoras de respuestas biológicas de cicatrización y regeneración tisular para ser incorporadas a un vehículo eficaz.

\section{Conclusión:}

Los resultados de este estudio preliminar demuestran que el Fluoruro di-amino de plata al $38 \%$ es capaz de penetrar gran parte de dentina afectada por caries, aumentando la dureza superficial de la lesión. El producto experimental a base de resina fluida modificada con nanopartículas de cinc aumenta la dureza en la superficie de las lesiones, aunque no es posible rastrear las partículas más allá de $15 \mu \mathrm{m}$ dentro del tejido afectado por caries.

\section{Agradecimientos:}

Los ensayos preliminares fueron realizados con el apoyo especializado de las Dras. Natalia Angel Villegas (Facultad de Ciencias Químicas, UNC), Verónica Brunetti y Eliana Farías (Departamento de Físico-Química, Facultad de Ciencias Químicas, UNC) y del Laboratorio LAMARX (Facultad de Matemática, Astronomía y Física, UNC).

\section{Bibliografía:}

1. Chen HF, Clarkson BH, Sunk, Mansfield JF. Self assembly of synthetic hydroxyapatite nanorods into enamel prism like structure. $\mathbf{J}$ Colloid Interface Sci 2005;288(1):97-103.

2. Burwell AK, Thula-Mata T, Gower LB, Habelitz S, Kurylo M, Ho SP, Chien YC, Cheng J, Cheng NF, Gansky SA, Marshall SJ, Marshall GW. Functional remineralization of dentin lesions using polymer-induced liquid- precursor process. PLoS One. 2012;7(6): e38852. doi: 10.1371/journal.pone.0038852.

3. Doméjean S, Ducamp R, Léger S, Holmgren C. Resin infiltration of non-cavitated caries lesions: a systematic review. Med Princ Pract. 2015;24(3):216-21. doi: 10.1159/000371709.

4. Silva LO, Signori C, Peixoto AC, Cenci MS, Faria-E-Silva AL. Color restoration and stability in two treatments for white spot lesions. Int J Esthet Dent. 2018;13(3):394403.

5. Osorio R, Cabello I, Medina-Castillo AL, Osorio E, Toledano M. Zinc-modified nanopolymers improve the quality of resindentin bonded interfaces. Clin Oral Investig. 2016 Dec;20(9):2411-2420. doi: 10.1007/s00784-016-1738-y

6. Najeeb S, Khurshid Z, Zafar MS, Khan AS, Zohaib S, Martí JM, Sauro S, Matinlinna JP, Rehman IU. Modifications in Glass Ionomer Cements: Nano-Sized Fillers and Bioactive Nanoceramics. Int J Mol Sci. 2016 Jul 14;17(7). pii: E1134. doi: 10.3390/ijms 17071134 .

7. Mei ML, Lo ECM, Chu CH. Arresting Dentine Caries with Silver Diamine Fluoride: What's Behind It? J Dent Res 2018 Jul;97(7):751-758. doi: $10.1177 / 0022034518774783$.

8. Oliveira BH, Rajendra A, Veitz-Keenan A, Niederman R. The Effect of Silver Diamine Fluoride in Preventing Caries in the Primary Dentition: A Systematic Review and MetaAnalysis. Caries Res. 2018 Jun 6;53(1):24-32. doi: $10.1159 / 000488686$.

9. Chu CH, Lo EC. Microhardness of dentine in primary teeth after topical fluoride applications. J Dent 2008; 36(6):387-391.

10. Marrese M, Guarino V, Ambrosio L. Atomic Force Microscopy: A Powerful Tool to Address Scaffold Design in Tissue Engineering. J Funct Biomater 2017, 8, 7; doi:10.3390/jfb8010007.

11. Zhang S. Fabrication of novel biomaterials through molecular self-assembly. Nat Biotechnol. 2003; 21:1171-1178.

12. Jaspart S, Piel G, Delattre L, Evrard B. Solid lipid microparticles: formulation, preparation, characterization, drug release and applications. Expert Opin Drug Deliv 2005; 2 : $1-13$.

13. 15-Manjunath K, Reddy JS, Venkateswarlu V. Solid Lipid Nanoparticles 
as drug delivery Systems. Methods Find Exp Clin Pharmacol 2005; 27: 1-20.

14. Sainz Aja M, Silvero MJ, Angel N, Molina GF, Palma SD. Plataformas nanotecnológicas para la infiltración de lesiones por caries. Libro de resúmenes del $50^{\circ}$ Encuentro Anual de la SAIO, San Nicolás (Bs. As.), noviembre de 2017, pp: 43.

15. Meyer-Lueckel H, Paris S. Infiltration of natural caries lesions with experimental resins differing in penetration coefficients and ethanol addition. Caries Res 2010; 44: 408414.

16. Lin LM, Ricucci D, Huang GT. Regeneration of the dentine-pulp complex with revitalization/revascularization therapy: challenges and hopes. Int Endod J. 2014 Aug;47(8):713-24. doi: 10.1111/iej.12210.

\section{Palabras claves:}

INFILTRACIÓN DE CARIES, RE MINERALIZACIÓN, NANOTECNOLOGÍA

\section{Keywords:}

CARIES INFILTRATION, REMINERALIZATION, NANOTECHNOLOGY

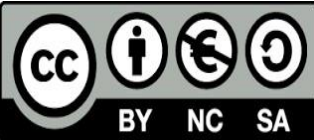

